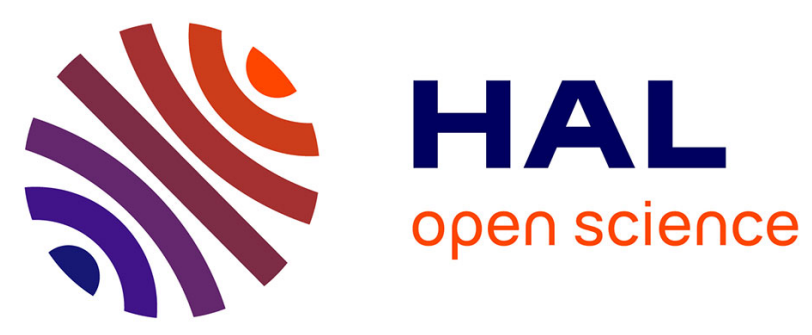

\title{
Trial Design to Estimate the Effect of Vaccination on Tuberculosis Incidence in Badgers
}

\author{
Inma Aznar, Guy Mcgrath, Denise Murphy, Leigh A.L. Corner, Eamonn \\ Gormley, Klaas Frankena, Simon J. More, Wayne Martin, James O'Keeffe, \\ Mart C.M. de Jong
}

\section{To cite this version:}

Inma Aznar, Guy Mcgrath, Denise Murphy, Leigh A.L. Corner, Eamonn Gormley, et al.. Trial Design to Estimate the Effect of Vaccination on Tuberculosis Incidence in Badgers. Veterinary Microbiology, 2011, 151 (1-2), pp.104. 10.1016/j.vetmic.2011.02.032 . hal-00701908

\section{HAL Id: hal-00701908 \\ https://hal.science/hal-00701908}

Submitted on 28 May 2012

HAL is a multi-disciplinary open access archive for the deposit and dissemination of scientific research documents, whether they are published or not. The documents may come from teaching and research institutions in France or abroad, or from public or private research centers.
L'archive ouverte pluridisciplinaire HAL, est destinée au dépôt et à la diffusion de documents scientifiques de niveau recherche, publiés ou non, émanant des établissements d'enseignement et de recherche français ou étrangers, des laboratoires publics ou privés. 


\section{Accepted Manuscript}

Title: Trial Design to Estimate the Effect of Vaccination on Tuberculosis Incidence in Badgers

Authors: Inma Aznar, Guy McGrath, Denise Murphy, Leigh A.L. Corner, Eamonn Gormley, Klaas Frankena, Simon J. More, Wayne Martin, James O'Keeffe, Mart C.M. De Jong

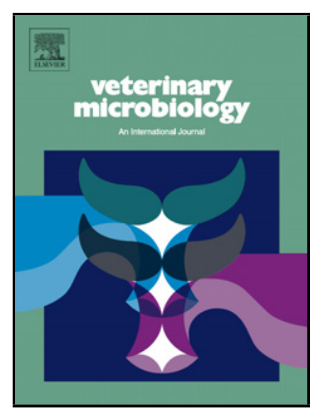

PII:

DOI: S0378-1135(11)00113-1

Reference: doi:10.1016/j.vetmic.2011.02.032

To appear in: $\quad$ VETMIC

Please cite this article as: Aznar, I., McGrath, G., Murphy, D., Corner, L.A.L., Gormley, E., Frankena, K., More, S.J., Martin, W., O'Keeffe, J., De Jong, M.C.M., Trial Design to Estimate the Effect of Vaccination on Tuberculosis Incidence in Badgers, Veterinary Microbiology (2010), doi:10.1016/j.vetmic.2011.02.032

This is a PDF file of an unedited manuscript that has been accepted for publication. As a service to our customers we are providing this early version of the manuscript. The manuscript will undergo copyediting, typesetting, and review of the resulting proof before it is published in its final form. Please note that during the production process errors may be discovered which could affect the content, and all legal disclaimers that apply to the journal pertain. 


\section{Trial Design to Estimate the Effect of Vaccination on Tuberculosis Incidence in Badgers}

3 Inma Aznar ${ }^{1 *}$, Guy McGrath ${ }^{1}$, Denise Murphy ${ }^{2}$, Leigh A.L. Corner ${ }^{3}$, Eamonn Gormley ${ }^{3}$,

4 Klaas Frankena ${ }^{4}$, Simon J. More ${ }^{1}$, Wayne Martin ${ }^{5}$, James O’Keeffe ${ }^{2}$, Mart C.M. De Jong ${ }^{4}$

$6{ }^{1}$ CVERA, Veterinary Science Centre, School of Agriculture Food Science and Veterinary

$7 \quad$ Medicine, University College Dublin, Belfield, Dublin 4, Ireland

$8 \quad{ }^{2}$ Department of Agriculture, Fisheries and Food (DAFF), Ireland

$9{ }^{3}$ Veterinary Science Centre, School of Agriculture Food Science and Veterinary Medicine,

10 University College Dublin, Belfield, Dublin 4, Ireland

$11{ }^{4}$ Quantitative Veterinary Epidemiology group, Wageningen Institute of Animal Sciences,

12 Wageningen University, P.O. Box 338, 6700 AH Wageningen, The Netherlands

$13{ }^{5}$ Department of Population Medicine, University of Guelph, Guelph, ON, Canada N1G2W1

15 *inma.aznar@ucd.ie

161 Abstract

18 The principal wildlife reservoir of Mycobacterium bovis in Ireland is the European badger.

19 Studies in the Republic of Ireland (RoI) have shown that badgers culled in association with cattle

20 herd tuberculosis (TB) breakdowns (focal culling) have a higher prevalence of infection than the

21 badger population at large. This observation is one rationale for the medium term national

22 strategy of focal badger culling. A vaccination strategy for the control of TB in badgers is a

23 preferred long-term option. The Bacillus Calmette-Guérin (BCG) vaccine has been shown to

24 decrease disease severity in captive badgers under controlled conditions. As the vaccine has been

25 tested in a controlled environment with precise information on infection pressure, it cannot be

26 assumed a priori that the effects of vaccination are similar in the wild, where other environmental 
27 and/or ecological factors prevail. For this reason we have designed a vaccine field trial to assess

28 the impact of vaccination on the incidence of TB infection in a wild badger population.

30 The selected study area for the vaccine trial (approximately 755 square kilometers) is divided into

31 three zones each of which has similar characteristics in terms of size, number of main badger

32 setts, cattle herds, cattle and land classification type. Three vaccination levels $(100 \%, 50 \%$ and

$330 \%$ ) will be allocated to the three zones in a way that a gradient of vaccination coverage North to

34 South is achieved. The middle zone (Zone B) will be vaccinated at a $50 \%$ coverage but Zone A

35 and $\mathrm{C}$ will be randomly allocated with $100 \%$ or $0 \%$ vaccination coverage. Vaccination within

36 Zone B will be done randomly at individual badger level.

38 The objective of this paper is to describe the design of a field TB vaccination trial for badgers, the

39 epidemiological methods that were used to design the trial and the subsequent data analysis. The

40 analysis will enable us to quantify the magnitude of the observed vaccination effect on M. bovis

41 transmission in badgers under field conditions and to improve our knowledge on the biological

42 effects of vaccination on susceptibility and infectiousness.

44 Keywords: Vaccine trial, badgers, BCG vaccine, Ireland, tuberculosis, M. bovis

\section{$47 \quad 2 \quad$ Introduction}

49 Ireland initiated an eradication program for bovine tuberculosis (bTB) as early as 1950 (More and

50 Good, 2006). The adopted test-and-slaughter policy achieved a 97\% reduction in cattle

51 tuberculosis prevalence, from $17 \%$ to $0.5 \%$ prevalence in the initial ten years (Watchorn, 1965). 
52 Since then, bTB prevalence has remained relatively unchanged despite the introduction of a range

53 of measures aimed at reducing cattle to cattle transmission (Griffin and Dolan, 1995).

55 In the 1970s in England, badgers were first suspected as a reservoir for Mycobacterium bovis 56 (Krebs, 1997). The first infected badger was discovered in Ireland in 1974 (Noonan et al., 1975).

57 Since then numerous papers have been published that confirm badgers as the main wildlife $M$.

58 bovis reservoir in Ireland and England (Barrow and Gallagher, 1981; Cheeseman et al., 1981;

59 Fagan, 1993; Gallagher et al., 1998). A recent study carried out in Ireland detected a prevalence

60 of $36.3 \%$ in badgers trapped as part of Department of Agriculture, Fisheries and Food (DAFF)

61 culling operations; the prevalence reported here was much higher than in previous studies where

62 less comprehensive bacteriological culture methods had been used (Murphy et al., 2010).

64 The precise role of badgers in the dynamics of bTB is not clear. Several studies in Ireland have 65 linked badger removal with a subsequent reduction in bTB incidence (Eves, 1999; Griffin et al., 66 2005; More and Good, 2006; Olea-Popelka et al., 2009). However, in a field trial carried out in

67 Britain, the reduction in cattle TB incidence in culled areas was only modest and an increase in 68 TB incidence, albeit transient (Jenkins et al., 2008), was observed in non culled neighboring areas

69 (Woodroffe et al., 2007). Pope et al. (2007) concluded that the increased prevalence observed in 70 neighboring areas was associated with medium and long-distance badger dispersal and 71 emphasized the importance of taking into account the potential negative effects associated with 72 badger dispersal when using culling as a disease control strategy. Although there are 73 discrepancies between different studies about the efficiency of badger culling in the control of 74 bTB, they all provide compelling evidence that badgers play an important role in the maintenance 75 of it. Therefore, addressing infection in badgers is considered vital when trying to control bovine 76 tuberculosis in the aforementioned countries. In the short- to medium-term in Ireland, focused 77 badger culling is being conducted as part of a broader national TB control programme, to limit 
78 TB maintenance in areas where TB incidence in cattle is high. However, badgers are a protective

79 species in the Republic of Ireland (ROI) under the 1976 Wildlife Act. Consequently, alternative

80 strategies to badger culling are being sought in the long term for the control of bovine

81 tuberculosis.

82

83 In 2001, a 10 year work program was designed in Ireland to study the possibility of using Bacillus

84 Calmette-Guérin (BCG) vaccine in badgers to assist in the control and eradication of TB in cattle.

85 The program consisted of a sequence of experimental studies carried out initially with captive

86 badgers (Corner et al., 2007; Lesellier et al., 2009). Although vaccines can be tested in a

87 controlled environment for evidence of both protection and decreased transmission, it cannot be

88 assumed $a$ priori that the effects of vaccination are similar in the wild where other factors may

89 play a role. For this reason, a vaccine field trial to assess the impact of vaccination on the

90 incidence of tuberculosis in a wild badger population has been designed as part of the ten year

91 project.

92

93 The objective of this paper is to describe a field trial design, the epidemiological methods that

94 were used to design the trial and the subsequent data analysis. The analysis will enable us to

95 quantify the magnitude of the observed vaccination effect on transmission under field conditions

96 and to improve our knowledge on the biological effects of the vaccination on susceptibility and

97 infectiousness of badgers.

98

993 Theoretical basis: a review

100

101

\subsection{Initial considerations in designing a vaccine trial}

102 
103 Specifying clear question(s) of interest is essential when designing a trial to evaluate the effects

104 of vaccination. Different vaccination programs have different aims; the question of interest could

105 vary, for example from how good vaccination is in protecting the individual against infection to

106 what reduction in infectiousness can be achieved. The effect of interest will determine the study

107 unit, parameters of effect, as well as the level of information required (Halloran et al., 1997). The

108 main question of interest in our study is to determine the efficiency of badger vaccination in

109 reducing $M$. bovis transmission. Specifically we are interested in the value of the reproduction

110 ratio $(\mathrm{R})$ at different levels of vaccination $(\mathrm{p})$; ie $\mathrm{R}(\mathrm{p})$. We will clarify how to estimate $\mathrm{R}(\mathrm{p})$

111 subsequently.

112

113 Vaccines that reduce transmission in a population can have a beneficial effect in vaccinated as

114 well as in unvaccinated individuals. Most studies of vaccine efficacy emphasize the direct benefit

115 of vaccination (often called 'protective ability') to vaccinated individuals. Following Halloran et

116 al. (1999), we denote this as $\mathrm{VE}_{\mathrm{S}}$ or Vaccine Efficacy for Susceptibility. In addition, there often

117 are indirect benefits for both vaccinated and unvaccinated individuals which Halloran et al.

118 denoted as Vaccine Efficacy for Infectiousness $\left(\mathrm{VE}_{\mathrm{I}}\right)$. These measures will be estimated in this

119 trial; however, for subsequent modelling purposes we will emphasize $\mathrm{R}(\mathrm{p})$

\subsection{Parameters of effect}

123 In order to estimate $\mathrm{R}(\mathrm{p})$, we define four transmission parameters:

124 - transmission from a vaccinated to a vaccinated badger $\left(\beta_{\mathrm{VV}}\right)$;

125 - transmission from a vaccinated to an unvaccinated badger $\left(\beta_{\mathrm{vU}}\right)$;

126 - transmission from an unvaccinated to a vaccinated ( $\left.\beta_{\mathrm{UV}}\right)$, and

127 - transmission from an unvaccinated to an unvaccinated badger $\left(\beta_{\mathrm{UU}}\right)$. 
129 The average number of secondary cases caused by one typically infected individual in a fully 130 susceptible population (Diekmann and Heesterbeek 2000) is called the Basic Reproduction Ratio $131\left(\mathrm{R}_{0}\right)$. Reproduction ratios of host populations in which interventions are taking place are often 132 represented by $R$ to distinguish them from $R_{0}$. The reproduction ratio as a function of the 133 proportion of vaccinated badgers $\mathrm{R}(\mathrm{p})$ can be determined from the transmission rates described 134 above and the time that infected badgers remain infectious $(\mathrm{T})$. Transmission rates are a 135 combination of the infectiousness of the donor and the susceptibility of recipient individuals, and

136 since vaccination with BCG has the potential to affect both of these, $\mathrm{R}(\mathrm{p})$ will be an important 137 parameter for understanding the impact of badger vaccination in disease transmission and 138 population dynamics of $M$. bovis. Ultimately, the R(p) obtained from the badger vaccine trial will 139 be used in further modelling aimed at assessing badger vaccination as a strategy to 140 control/eradicate $M$. bovis infection in cattle.

142 As noted above, vaccine efficacy/effectiveness has been traditionally defined as 1 minus some 143 measure of relative risk (RR) of the incidence of disease in the vaccinated group compared to the 144 incidence in the non-vaccinated group (Halloran et al., 1999). In the past, the main objective of 145 human vaccine studies was to measure individual protection against infection or disease $\left(\mathrm{VE}_{\mathrm{S}}\right)$. 146 Perhaps less appreciated, but not less important, is the ability of a vaccine to reduce the duration 147 or severity of the infectiousness of those vaccinates that become infected ( $\left.\mathrm{VE}_{\mathrm{I}}\right)$ (Longini et al, 148 1998). The latter effect has been reported in vaccine studies using BCG vaccine by the 149 subcutaneous or mucosal routes in badgers (Corner et al., 2008). In these experiments, M. bovis 150 was recovered from both vaccinated and non-vaccinated badgers after being challenged with the 151 mycobacterium; however, a reduction in the size, number and distribution of gross and 152 histological lesions in vaccinated badgers compared to non-vaccinated badgers was demonstrated.

153 Vaccination did not confer individual protection against infection in the mentioned study, but this 
154 has to be cautiously interpreted as vaccine protection could be dependent on the infection dose. It

155 is not known what the infectious dose is in natural infections. In field vaccine trials in possum in

156 New Zealand, using conjunctival and intranasal vaccination (Corner et al., 2002) and more

157 recently vaccination by oral delivery (Tompkins et al., 2009), significant protection against

158 natural exposure was seen in the vaccinated group compared to the control groups. Protection was

159 much higher than predicted from previous studies where possums had been experimentally

160 challenged (Corner et al., 2001; Buddle et al., 2006).

161 Estimation of $\mathrm{R}(\mathrm{p}), \mathrm{VE}_{\mathrm{S}}$ and $\mathrm{VE}_{\mathrm{I}}$ will give us a more detailed understanding of the ways BCG

162 vaccine works in a wild badger population.

163

1644 Epidemiological contribution to the design of the vaccine trial

$165 \quad 4.1$ Study site

166

167 Prior to deciding on our study site for the badger vaccine trial, several epidemiological and 168 logistic factors were considered:

169 - Culling history. Because of the badger culling pollicy in Ireland, it was important to 170 have confidence that the area selected for the trial had been protected from culling 171 for some time before the commencement of the trial. Badger culling in that area 172 could have had a negative effect not just in the total number of individuals captured 173 but also on the initial prevalence of TB among badgers.

174 - Knowledge of sett location. Knowledge of the area in terms of sett location was 175 considered an advantage and helped in dividing the study area into three similar 176 zones (see below). 
- Community and technical support. Good support from the local farming community as well as from from both the District Veterinary Offices (DVOs) and Regional Veterinary Laboratories (RVLs) was vital.

181 (Figure 1). The size of the area is approximately 755 square kilometers. This area had been part 182 of one of the reference areas in the Four Area Project (FAP) (Griffin et al., 2005) and will have 183 been protected from culling for at least two years before the start of the vaccine trial. A 184 prevalence of infection of $30 \%$ is expected based on historical data from neighbouring areas.

\subsection{Trial design}

188 A vaccine trial to exclusively determine $\mathrm{VE}_{\mathrm{S}}$ can be designed by vaccinating one or several 189 populations with the same vaccination coverage, with coverage being strictly inferior to $100 \%$ $190(40 \%, 50 \%, 60 \%$, etc) so that a number of infections within the vaccinated group can occur.

191 Because we aimed to estimate both $\mathrm{VE}_{\mathrm{S}}$ and $\mathrm{VE}_{\mathrm{I}}$, then two populations vaccinated with different

192 vaccination coverage are required (Longini et al., 1998). In this paper it is explained how the

193 vaccination fractions for these two populations have to be selected to maximise the differences on

194 indirect effects between the two populations. This can be achieved with one population

195 vaccinated at $0 \%$ and the other vaccinated at the highest percentage that allows a minimum

196 number of infections to happen (critical vaccination fraction). Likewise to estimate the four betas

197 (transmission rates), different levels of coverage are needed (DeJong et al., 1996).

199 Our objectives could have therefore been attained with two populations, one vaccinated at $0 \%$ 200 and the other at $100 \%$ vaccination coverage (badger trapping rates will never be $100 \%$ effective; 201 also the dynamics of the badger population ensures a certain number of susceptible badgers every 
202 year and therefore a certain number of infections). We chose though to include a third population

203 vaccinated at $50 \%$ to optimise the design in two ways: firstly by making sure that there will be

204 enough new infections even if the "100\%" vaccination would lead to $\mathrm{R}<1$ and secondly by

205 allowing us to estimate the area effect (in the case of only two populations, the parameters would

206 be estimated from different populations and it would not be possible to disentangle the area

207 effect).

209 The trial area is divided into three zones with similar characteristics in terms of size, number of

210 main badger setts, cattle herds, cattle and land classification (Figure 2). In these zones,

211 vaccination coverage will be of allocated 100, 50 and $0 \%$, with the gradient of coverage (either

$212100 \%$ to $0 \%$ from north to south, or vice-versa) being allocated randomly at the start of the trial.

213 Badger data were acquired through rigorous surveying of the entire study area. Previous

214 surveying had been performed in sections of the study area as part of the FAP reference area and

215 through the activities of the DAFF's licensed badger culling policy on setts adjacent to herd bTB

216 breakdowns. Setts previously recorded were revisited during the recent survey and assessed for

217 signs of activity. All bovine data were derived from the Animal Health Computer System

218 (AHCS) and farm outlines were taken from the Land Parcel Identification System (LPIS). Land

219 use classifications were defined using the CORINE dataset (Coordination of Information on the

220 Environment, 2000). Using ArcMap 9.2 (ESRI, Redlands, CA, USA) with geo-rectified colour

221 orthophotography and vectorized 1:50,000 data (Ordnance Survey Ireland, Dublin, Ireland),

222 natural boundaries, where possible, were selected to define the perimeter and internal boundaries

223 of the study area. The aim was to achieve similar infection pressure from cattle and badgers in the

224 three zones.

225

226 Badgers will be captured, treated with a vaccine or a placebo depending on the randomly

227 allocated treatment and then released. In zone B (with 50\% vaccine coverage), each badger at 
228 first capture will be randomly allocated to either vaccine or placebo. In each zone throughout the

229 study period, the treatment will be administered every year to avoid possible waning of vaccine

230 effects. Live M. bovis BCG strain Danish will be used. It will be prepared in a lipid formulation

231 for oral delivery, containing $10^{8}$ colony forming units/ml. A lipid-only placebo with identical

232 visual characteristics, texture and viscosity to the vaccine, and in identical syringes, will also be

233 used (Aldwell et al., 2003a: Aldwell et al., 2003b). Vaccine and placebo control samples will be

234 coded at the laboratory where they are prepared, and neither field staff nor data analysts will be

235 aware of the vaccine status of individual animals.

237 The trial will employ a capture-tag-release regime with both cages and stopped wire restraints

238 being used. Badgers in the trial will be 'hand vaccinated'; that is, each animal will be individually

239 orally dosed with the vaccine. Each badger will be permanently identified with a tattoo and

240 passive transponder (microchip) when first captured. At each capture, badgers will be examined

241 and a blood sample collected. Humoral immune responses (serologic titres) will be used to

242 determine the badger's infection status and to detect a change in infection status; that is, to detect

243 both pre-existing infection in badgers as they are recruited to the study and the occurrence of new

244 infections on recapture. Key data, including sex, estimated age (cub, juvenile, and yearling, adult

245 and old adult), body weight, presence of injuries and the GPS location of the cage trap or

246 restraint, will be recorded at each badger capture. All data collected in the field will be recorded

247 onto handheld computers. The trial will last four years and there will be two 'catching' sweeps of

248 the entire area each year. At the end of the trial, badgers in the three zones will be depopulated

249 and a detailed post mortem examination will be conducted on all badgers, involving an

250 examination for gross pathology and the collection of samples for histopathology and

251 bacteriology. The severity of infection will be assessed from the number, distribution and the

252 severity of gross lesions, the number and distribution of histological lesions, and the number and

253 distribution of culture positive tissues and the bacterial load in those tissues. 
255 Badger removal will be undertaken within the trial area when three or more standard reactors are

256 disclosed in a herd only if active badger setts are found in the farm and all other sources of

257 infection (residual, purchase and farm to farm spread) have been ruled out by an epidemiological

258 investigation. If culling of badgers is deemed necessary for control of tuberculosis in cattle herds,

259 the culling will be carried out by field staff of the project when they next trap in the designated

260 area.

2625 Analysis of the vaccine trial data

263

$264 \quad 5.1$ General description

266 The outcome from the vaccine trial will be in the form of a Bernoulli experiment: as badgers are

267 trapped and a blood sample taken, we will gather information on whether these badgers have

268 become or "are" infected (assigned value 1) or not (assigned value 0) during the time at risk (time

269 between two subsequent trappings). Infection in this case will be defined by serologic results i.e.

270 by sero-positivity. Records on individual badgers will be taken at each successful capture (not

271 necessarily at each trapping exercise) such as location of the badger at the time of the trapping

272 (zone A, B or C) and its vaccination status. Other demographic data will be also recorded.

274 From the observations at each subsequent capture of each individual badger, the following 275 variables will be extracted:

277 - Time interval $(\Delta \mathrm{t})$ between the two captures 
- Disease status of the badger at the beginning and at the end of the time interval

279 - Vaccination status of the badger

- Zone where the animal was observed at the beginning and end of the time interval (A, B or C)

- Average prevalence of infection in the zone where the badger was caught during $\Delta t$ (PrevA, PrevB, PrevC)

- Average fraction of infected badgers that are vaccinated during $\Delta t$ in the zone where the

287 The rate at which vaccinated/non-vaccinated badgers acquire infection from vaccinated and non288 vaccinated badgers $\left(\beta_{\mathrm{VV}}, \beta_{\mathrm{VU}}, \beta_{\mathrm{UV}}, \beta_{\mathrm{UU}}\right)$ can be estimated based on the observed probability of 289 becoming infected for each of these individuals. The estimated $\beta$ s will be used to calculate $\mathrm{VE}_{\mathrm{S}}$,

$290 \mathrm{VE}_{\mathrm{I}}$ and the reproduction ratio as a function of the vaccination coverage $(\mathrm{R}(\mathrm{p}))$.

\subsection{Estimation of the transmission parameters}

294 The rate at which vaccinated/non-vaccinated badgers acquire infection from vaccinated and non-

295 vaccinated badgers $\left(\beta_{\mathrm{VV}}, \beta_{\mathrm{VU}}, \beta_{\mathrm{UV}}, \beta_{\mathrm{UU}}\right)$ can be estimated based on the observed probability of 296 becoming infected for each of these individuals. For the purposes of explanation, we shall at first 297 ignore the vaccination state of the badgers. A stochastic susceptible-infectious (SI) model can be 298 used then to describe the transmission of M. bovis in the trial where transmission can occur if an 299 infectious and a susceptible individual make contact. The number of infectious contacts (NIC, 300 contacts with an infectious individual) encountered by each randomly chosen susceptible 301 individual in a period of time $\Delta t$ can be expressed as: 


$$
N I C=\beta *(I / N) * \Delta t
$$

304 Where:

305

$306 \beta=$ Transmission parameter

$307 \Delta \mathrm{t}=$ Time interval

308 I= Number of infected individuals*

$309 \mathrm{~N}=$ Total number of individuals

$310 \mathrm{I} / \mathrm{N}=$ Prevalence of infected badgers*

$311 *$ For our purpose infected badgers are deemed to be infectious

313 From the above it can be derived that the number of 'successful' infectious contacts (infectious

314 contacts that result in transmission events) encountered by this randomly chosen individual in a

315 period of time $\Delta \mathrm{t}$ follows a Bernoulli distribution with probability:

$$
p=1-e^{-\beta * I * \Delta t / N}
$$

319 The observed number of new infections (C) among all susceptible badgers at the end of the time

320 interval between two captures can then be modelled using a binomial distribution where $\mathrm{S}$ is the

321 number of susceptible badgers at the beginning of the time interval and the probability that each

322 of these badgers will become infected during that time interval is defined by $1-e^{-\beta * I * \Delta t / N}$

$$
E(C)=S *\left(1-e^{-\beta * I * \Delta t / N}\right)
$$


326 Based on serologic results we will be able to determine the number of new cases among the

327 susceptible badgers (C). By using a Generalized Linear Model (GLM) with a complementary-log-

$328 \log$ link function, a binomial error function, with binomial total $\mathrm{S}$ and offset $\ln ((I / N) * \Delta t)$, the

329 transmission parameter $\beta$ can be estimated.

330

331 Following the introduction of the vaccination protocol, there will be heterogeneity in the

332 vaccination status of badgers; we will have four different $\beta$ values with two sub indexes, of the

333 type $\beta_{\mathrm{vu}}$. The first sub index will refer to the vaccination state of the infectious badger and the

334 second sub index to the vaccination state of the susceptible animal (to which the first has made

335 contact). Clearly, we know the vaccination status of the receiving susceptible badger and thus we

336 can calculate $\beta_{\text {.u }}$ and $\beta_{\text {.v }}$ separately. However to account for donor (infectious) badger, we use the

337 differences in the fraction of infected badgers that are vaccinated in the different zones.

338

339 Let's assume that a badger that was originally trapped in zone A at time ' $t$ ' is trapped again in

340 zone A at time ' $t+1$ ' and that this badger had been vaccinated. Vaccination coverage in zone A is

341 targeted to be $100 \%$, but since not all badgers will be trapped in each trapping exercise it will take

342 2-3 trapping rounds to approach this goal. Our hypothetical badger will then have infection

343 pressure coming from both vaccinated and non-vaccinated infected badgers. The number of

344 infectious contacts encountered by this randomly selected badger in that period of time is defined

345 by:

$$
N I C=\left(\beta_{V V} * I_{V} / N+\beta_{U V} * I_{U} / N\right) * \Delta t
$$

349 If we assume that there is a multiplicative effect on NIC as the fraction of infected vaccinated

350 badgers increases, after doing some algebraic manipulations (De Jong et al., 1996): 


$$
N I C=e^{\beta_{V V} * F_{V A}+\beta_{U V} *\left(1-F_{V A}\right)} * \operatorname{Prev} A * \Delta t
$$

354 or:

$$
N I C=e^{\beta_{U V}+\left(\beta_{V V}-\beta_{U V}\right) * F_{V A}} * \operatorname{PrevA} * \Delta t
$$

358 where $\mathrm{F}_{\mathrm{VA}}$ is the fraction of infected badgers that are vaccinated in zone $\mathrm{A}$ and PrevA is the average prevalence of infection in zone A during $\Delta t$. If we set $\beta_{U V}=K_{0}$ and $\left(\beta_{V V}-\beta_{U V}\right)=K_{1}$ then we can write:

$$
E\left(C_{V}\right)=S *\left(1-e^{-e^{\left(K_{0}+K_{1} * F_{V A}\right)} * \operatorname{Pr} e v A * \Delta t}\right)
$$

364 Since we will know the observed infection status of the specific badger $\left(\mathrm{C}_{\mathrm{V}}\right)$ at the end of the time 365 interval $(\Delta \mathrm{t})$, we can fit a GLM with $\log \left(\operatorname{PrevA} \Delta \mathrm{t}\right.$ ) as an offset and calculate $\mathrm{K}_{0}$ and $\mathrm{K}_{1}$. We can 366 subsequently calculate $\beta_{\mathrm{UV}}$ and $\beta_{\mathrm{VV}}$ as:

$368 \quad \beta_{\mathrm{UV}}=\operatorname{Exp}\left[\mathrm{K}_{0}\right]$

$369 \quad \beta_{\mathrm{VV}}=\operatorname{Exp}\left[\mathrm{K}_{0}+\mathrm{K}_{1}\right]$

370

371 If we apply the same logic to model the observed infectious status of an unvaccinated badger $\left(\mathrm{C}_{\mathrm{U}}\right)$

372 that was trapped in zone $A$ at time $t$ and at time $t+1$, we could estimate $\beta_{\mathrm{UU}}$ and $\beta_{\mathrm{VU}}$ as:

373

374

$$
E\left(C_{U}\right)=S *\left(1-e^{-e^{\left(k_{0}+k_{1} * F_{V A}\right)} * \operatorname{Pr} e v A * \Delta t}\right)
$$


376 where $\beta_{\mathrm{UU}}=\operatorname{Exp}\left[\mathrm{k}_{0}\right]$ and $\beta_{\mathrm{VU}}=\operatorname{Exp}\left[\mathrm{k}_{0}+\mathrm{k}_{1}\right]$

378 This way we can estimate the four betas based on incidence data from all three zones. In zone C

379 there will be no (or very few) vaccinated badgers so $\mathrm{F}_{\mathrm{VC}}$ will be zero and that zone does not

380 contribute to the estimation of $\mathrm{k}_{1}$ and thus that area does not give information for $\beta_{\mathrm{vu}}$.

382 Vaccine Efficacy for Susceptibility can be calculated then as:

$$
V E_{S}=1-\left(\beta_{U V} / \beta_{U U}\right)
$$

We can also calculate Vaccine Efficacy for Infectiousness as:

$$
V E_{I}=1-\left(\beta_{V U} / \beta_{U U}\right)
$$

390 Finally we can estimate $\mathrm{R}(\mathrm{p})$ where $\mathrm{p}$ is the proportion of vaccinated as:

$$
\left.R(p)=\frac{1}{2} *\left[(1-p) * \beta_{U U}+p^{*} \beta_{V V}+\sqrt{\left((1-p) * \beta_{U U}+p^{*} \beta_{V V}\right)^{2}+4 * p *(1-p) * \beta_{U V} * \beta_{V U}}\right)\right] * T
$$

395 where $\mathrm{T}$ is the duration of the "infectious" period of a typical infected individual (for relative 396 comparisons of $\mathrm{R}(\mathrm{p})$ knowledge of $\mathrm{T}$ is not essential). 
397 For decision making, if $\mathrm{p}=0$ and $\beta_{\mathrm{UU}} \mathrm{T}<1$, then no vaccination is needed; if $\mathrm{p}=1$ and $\beta_{\mathrm{VV}} \mathrm{T}>1$,

398 vaccination will not stop spread of the disease. Otherwise, the vaccination fraction necessary in

399 order to achieve $\mathrm{R}(\mathrm{p})<1$ can be calculated.

400

4016 Concluding remarks

402

403 The aim of this paper was to present the theory applied to the design of a badger vaccine trial, as

404 well as the epidemiological methodology and statistical analysis that will help to interpret the 405 results obtained.

406

407 The vaccine trial area was divided into three zones A, B and C, such that differences in infection 408 pressure in the three zones at the beginning of the trial will be minimized. However, there is an 409 inherent assumption that the contact patterns between badgers will be similar in the three zones.

410 We believe that this is a reasonable assumption as the size of the total area is sufficiently small,

411 and the landscape and distribution of setts is very similar in the three zones. Further, changes with

412 respect to infection pressure in the three zones will occur as the vaccine starts working. Our

413 model is designed to adjust for these changes. For simplicity, we have only presented the analysis

414 relevant to badgers repeated trapped in the same zone. However, the final model can be modified

415 to accommodate other scenarios where badgers move from one zone to another.

417 Where possible, natural boundaries are used to define the perimeter of the study area. The internal 418 boundaries between the three zones include streams, rivers and roads. The external boundaries, 419 while not bio-secure, should be substantial enough to delineate badger territories. This would 420 reduce the extent of typical badger movement into and out of the study area. 
422 Defining specific questions of interest in the early stages of the vaccine trial was considered

423 crucial. Estimation of the reproduction ratio $(\mathrm{R}(\mathrm{p}))$ calculated as a function of the vaccination

424 coverage (p) will give us invaluable information on the impact of vaccination in disease

425 transmission and dynamics of $M$. bovis infection in badgers. The importance of considering the

426 potential indirect effects associated with the vaccination program in badgers has been highlighted

427 in the paper; furthermore, it has been shown how to estimate $R(p), V_{S}$ and $V E_{I}$ by using

428 different vaccination coverages in the study area.

430 Although the vaccine trial will not determine whether bTB can be eradicated in cattle and badgers

431 through a strategy of badger vaccination, the parameters obtained in the vaccine trial will be used

432 in a mathematical model of bTB transmission (currently under development) in order to assess

433 different control and eradication options for bTB in cattle in the Republic of Ireland.

7 Conflict of Interest Statement

437 The authors have not declared any conflict of interest.

440 Aldwell, F. E., Keen, D.L., Parlane, N.A., Skinner, M.A., de Lisle, G.W., Buddle, B.M., 2003a. Oral vaccination with Mycobacterium bovis BCG in a lipid formulation induces resistance to pulmonary tuberculosis in brushtail possums. Vaccine $22,70-76$.

443 Aldwell, F.E., Tucker, I.G., de Lisle, G.W., Buddle, B.M., 2003b. Oral delivery of 444 Mycobacterium bovis BCG in a lipid formulation induces resistance to pulmonary 445 tuberculosis in mice. Infection and Immunity 71, 101-108. 
Barrow, P.A., Gallagher, J., 1981. Aspects of the epidemiology of bovine tuberculosis in badgers and cattle. I. The prevalence of infection in two wild animal populations in south-west

Buddle, B.M., Aldwell, F.E., Kee, D.L., Parlane, N.A., Hamel, K.L., de Lisle, G.W., 2006. Oral vaccination of brushtail possums with BCG: investigation into factors that may influence vaccine efficacy and determination of duration of protection. N.Z. Vet. J. 54, 224-230.

Cheeseman, C.L., Jones, G.W., Gallagher, J., Mallinson, P.J., 1981. The population structure, density and prevalence of tuberculosis (Mycobacterium bovis) in badgers (Meles meles)

Corner, L.A.L., Norton, S., Buddle, B.M., Morris, R.S., 2002. The efficacy of bacille from four areas in south-west England. J. Appl. Ecol. 18, 795-804.

Corner, L.A.L., Buddle, B.M., Pfeiffer, D.U., Morris, R.S., 2001. Aerosol vaccination of the brushtail possum (Trichosurus vulpecula) with bacille Calmette-Guérin: the

Corner, L.A.L., Costello, E., Lesellier, S., O'Meara, D., Sleeman, D.P., Gormley, E., 2007. Experimental tuberculosis in the European badger after endobronchial inoculation of Calmette-Guérin vaccine in wild brushtail possums (Trichosurus vulpecula). Res. duration of protection. Vet. Micro. 81, 181-191. Mycobacterium bovis: I. Pathology and bacteriology. Res. Vet. Sci. 83, 53-62.

Corner, L.A., Costello, E., Lesellier, S., O'Meara, D., Gormley, E., 2008. Vaccination of European badgers (Meles meles) with BCG by the subcutaneous and mucosal routes induces protective immunity against endobronchial challenge with Mycobacterium bovis. Tuberculosis (Edinb) 88, 601-609.

De Jong, M.C.M., van der Poel, W.H.M., Kramps, J.A., Brand, A. and van Oirschot, J.T., 1996. Quantitative investigation of population persistence and recurrent outbreaks of bovine respiratory syncytial virus on dairy farms. Am. J. Vet. Res. 57, 628-633. 
471 Diekmann, O., Heesterbeek, J.A.P., 2000. Mathematical epidemiology of infectious diseases: model building, analysis and interpretation. Wiley series in mathematical and computational biology. Chichester, UK: Wiley.

474 Eves, J.A., 1999. Impact of badger removal on bovine tuberculosis in east County Offaly. Irish Vet. J. 52, 199-203.

Fagan, J., 1993. Tuberculosis in badgers in Ireland: Pathology. In: The badger. Proceedings of a seminar held on 6-7 March 1991 pp. 117-122.

Gallagher, J., Monies, R., Gavier-Widen, M., Rule, B., 1998. Role of infected, non-diseased badgers in the pathogenesis of tuberculosis in the badger. Vet. Rec. 142, 710-714.

Griffin, J.M., Dolan, L.A., 1995. The role of cattle-to-cattle transmission of Mycobacterium bovis in the epidemiology of tuberculosis in cattle in the Republic of Ireland: a review. Irish Vet. J. 48, 228-234.

Griffin, J.M., Williams, D.H., Kelly, G.E., Clegg, T.A., O'Boyle, I., Collins, J.D., More, S.J., 2005. The impact of badger removal on the control of tuberculosis in cattle herds in Ireland. Prev. Vet. Med. 67, 237-266.

Halloran, M.E., Struchiner, C.J., Longini, I.M., Jr., 1997. Study Designs for Evaluating Different Efficacy and Effectiveness Aspects of Vaccines. Am. J. Epidemiol. 146, 789-803.

Halloran, M.E., Longini, I.M., Jr., Struchiner, C.J., 1999. Design and Interpretation of Vaccine Field Studies. Epidemiol. Rev. 21, 73-88.

Jenkins, H.E., Woodroffe, R., Donnelly, C.A., 2008. The effects of annual widespread badger culls on cattle tuberculosis following the cessation of culling. Int. J. Infect. Dis. 12, Hewinson, R.G., Chambers, M., Gormley, E., 2009. Immunological responses and 
protective immunity in BCG vaccinated badgers following endobronchial infection with Mycobacterium bovis. Vaccine 27, 402-409.

Longini, I.M., Jr., Sagatelian, K., Rida, W.N., Halloran, M.E., 1998. Optimal vaccine trial design when estimating vaccine efficacy for susceptibility and infectiousness from multiple populations. Stat. Med. 17, 1121-1136.

More, S.J., Good, M., 2006. The tuberculosis eradication programme in Ireland: a review of scientific and policy advances since 1988. Vet. Microbiol. 112, 239-251.

Murphy, D., Gormley, E., Costello, E., O'Meara, D., Corner, L.A.L., 2010. The prevalence and distribution of Mycobacterium bovis infection in European badgers (Meles meles) as determined by enhanced post mortem examination and bacteriological culture. Res. Vet. Sci $88,1-5$.

Olea-Popelka, F.J., Fitzgerald, P., White, P., McGrath, G., Collins, J.D., O'Keeffe, J., Kelton, subsequent risk of bovine tuberculosis in cattle herds in county Laois, Ireland. Prev. Med.

514 Pope, L.C., Butlin, R.K., Wilson, G.J., Woodroffe, R., Erven, K., Conyers, C.M., Chris, M., Vet. $88,178-184$.

518 Tompkins, D.M., Ramsey, D.S.L., Cross, M.L., Aldwell, F.E., de Lisle, G.W., Buddle, B.M., Franklin, T., Delahay, R.J., Cheeseman, C.L., Chris, L., Burke, T., 2007. Genetic evidence that culling increases badger movement: implications for the spread of bovine tuberculosis. Mol. Ecol. 16, 4919-4929. 2009. Oral vaccination reduces the incidence of tuberculosis in free-living brushtail possums. Proc. R. Soc. B 276, 2987-2995.

Woodroffe, R., Gilks, P., Johnston, W.T., Le Fevre, A.M., Cox, D.R., Donnelly, C.A., Bourne, F.J., Cheeseman, C.L., Gettinby, G., MCinerney, J.P., Morrison, W.I., 2007. Effects of 
523 culling on badger abundance: implications for tuberculosis control. Journal of Zoology, $524 \quad 28-37$.

525 Watchorn, R.C., 1965. Bovine tuberculosis eradication scheme 1954-1965. Department of 526 Agriculture and Fisheries. Dublin. 


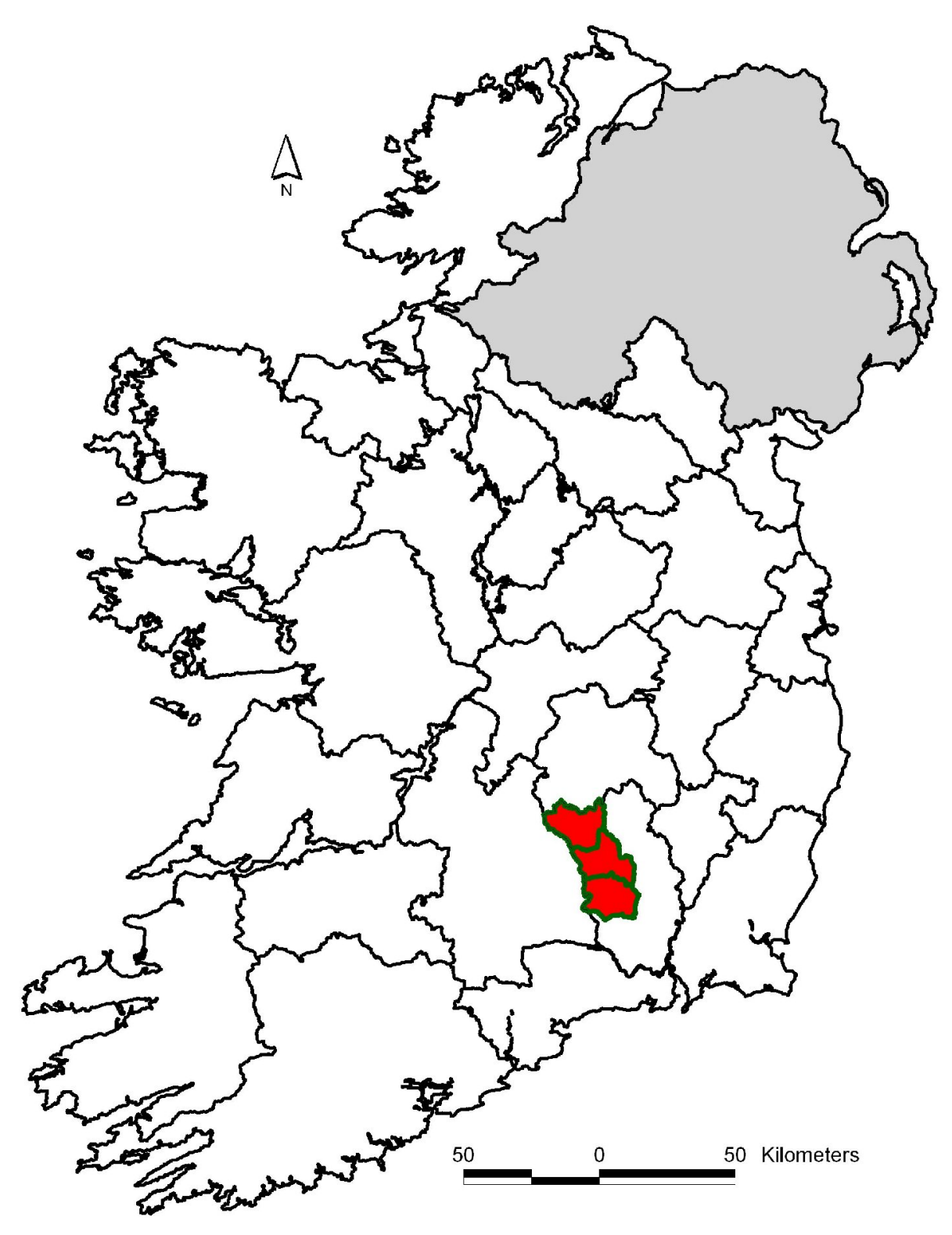

545

Figure 1. Study site selected for the vaccine trial is highlighted in red 

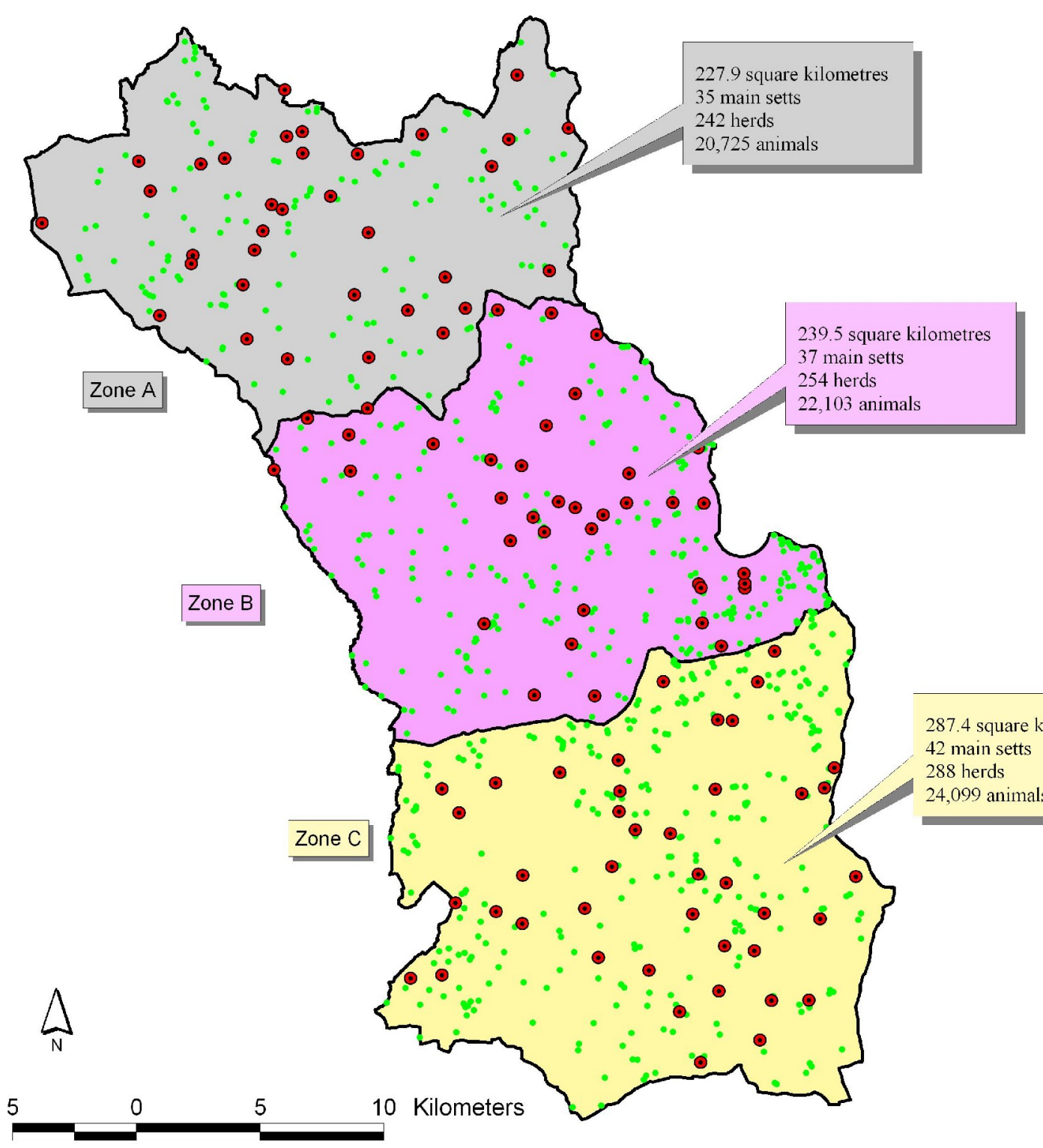

287.4 square kilometres 42 main setts 288 herds

568 Figure 2. Map showing study area divided into three zones A, B and C (grey, pink and yellow

569 respectively) where vaccination coverage will be of 100, 50 and $0 \%$. The gradient of coverage 
570 (100\% to $0 \%$ from north to south, or vice-versa) will be allocated randomly at the start of the

571 trial. Main badger setts are represented with red dots and green dots represent other sett types

572 